\title{
Quantitative Analysis of Brain White Matter Lesions in Multiple Sclerosis Subjects: Preliminary Findings
}

\author{
C. P. Loizou, Member, IEEE, C. S. Pattichis, Senior Member, IEEE, \\ I. Seimenis, E. Eracleous, C. N. Schizas, Senior Member, and M. Pantziaris
}

\begin{abstract}
In this study the value of magnetic resonance image (MRI) shape and texture analysis was assessed in multiple sclerosis (MS) subjects, both in differentiating between normal and abnormal tissue and in assessing disease progression. Shape and texture analysis was carried out in normal and diseased lesions in transverse sections of T2weighted magnetic resonance (MR) images acquired from 10 symptomatic untreated subjects with clinically isolated syndrome (CIS) scanned twice, with an interval of 6-12 months. All detected brain lesions were manually segmented by an experienced neurologist and confirmed by a neuro-radiologist, whilst different shape and texture features were extracted from the segmented lesions. The results showed that there was no significance difference between shape features of 0 and 6-12 months. For some texture features there was significance difference between normal tissue and MS lesions at 0 and 6-12 months and between MS lesions at 0 and 6-12 months (i.e contrast, difference variance, difference entropy, and other). Further research with more subjects is required for computing shape and texture features that may provide information for differentiating between normal tissue and MS lesions as well as for longitudinal monitoring of these lesions. In addition the proposed methodology can be used for the assessment of subjects at risk of developing future neurological events. The extracted shape and features can also offer additional information of undiagnosed lesions.
\end{abstract}

\section{INTRODUCTION}

$\mathrm{M}$ ultiple Sclerosis (MS) is a chronic idiopathic disease that results in multiple areas of inflammatory demyelination within the central nervous system. Within individuals the clinical manifestations are unpredictable, particularly with regard to the development of disability [1]. In [2] correlations between MS and disability were investigated, whereas in [3] disease subgroups were classified based on their MS disease severity.

Texture features quantify macroscopic lesions and also the macroscopic abnormalities that may be undetectable using conventional measures of lesion volume and number [1]. Texture analysis is used widely in magnetic resonance

Manuscript received March 17, 2008, accepted April 4, 2008.

C. P. Loizou is with the Department of Computer Science and Engineering, Intercollege, P.O. Box 51604, 3507 Limassol, Cyprus, 357-25381180; e-mail: loizou.c@ lim.intercollege.ac.cy.

C. S. Pattichis and C.N. Schizas, are with Department of Computer Science, University of Cyprus, Nicosia, Cyprus. (e-mail: pattichi, schizas@ucy.ac.cy).

I. Seimenis, and E. Eracleous, are with the Medical Diagnostic Center "Ayios Therissos", P.O. Box 28405, 2033 Nicosia, Cyprus (e-mail: yseimen@phys.uoa.gr, elenerac@logosnet.cy.net).

M. Pantzaris is with the Cyprus Institute of Neurology and Genetics, Nicosia, Cyprus, (e-mail: pantzari@cing.ac.cy). imaging (MRI) enabling disease characterization and quantification of disease distribution. The characterization of healthy and pathological lesions by using texture analysis in brain was investigated by [4]. Significant differences in texture between normal and disease spinal cord MS patients were found in [5]. Also the classification of active and nonactive lesions in MS patients from brain MRI was investigated in [6]. In [7] the performance of texture analysis and tissue discrimination between MS lesions and normal appearing white matter (NAWM) and normal white matter (NWM) was investigated for supporting early diagnosis in MS. In [8], texture features extracted from the brain of normal and Alzheimer diseased patients were found to be significantly different and that the progression of the disease is reflected in these measures.

The objective of this study was to assess the value of MRI shape and texture analysis in the study of MS brain lesions both in differentiating between normal and abnormal tissue and in assessing disease progression. The paper is organized as follows: In section II, materials and methods are given, in section III the results are presented, and finally section IV give the discussion and the concluding remarks.

\section{MAtERIALs AND MethodS}

\section{A. Acquisition of MR Images}

In agreement with the Cyprus national bioethics committee rules on clinical trials, ten subjects ( 7 male and 3 female), aged $30.5 \pm 11.6$ (mean age \pm standard deviation), with a clinical isolated syndrome (CIS) of MS and MRI detectable brain lesions were scanned twice with an interval of 6-12 months. All subjects were untreated and clinically examined by the neurologist following the MRI, and were given a disability score using the expanded disability status scale (EDSS) [9]. The EDSS scores for each patient are shown in Table I. Additionally 10 healthy, age-matched (mean \pm SD: $30.8 \pm 7.6)$ volunteers (4 male, 6 female) were scanned for image texture analysis on healthy brain parenchyma (NWM).

The magnetic resonance (MR) images were acquired using a $1.5 \mathrm{~T}$ whole body Philips ACS NT MR imager (Philips Medical Systems, Best, the Netherlands). A built-in quadrature radiofrequency (RF) body coil and a quadrature $\mathrm{RF}$ head coil were used for proton excitation and signal detection respectively. The images used for analysis were obtained using a T2-weighted turbo spin echo pulse sequence $(\mathrm{TR}=4408 \mathrm{~ms}, \mathrm{TE}=100 \mathrm{~ms}$, echo spacing $=10.8 \mathrm{~ms})$. 
TABLE I

Shape Features Statistical Analysis For The Lesions Detected Within Each Subject at 0 And 6-12 Months. The ManN-Whitney Rank Sum Test Shows With S AND NS the Features That ARe AND ARE Not Significantly Different Between 0 AND 6-12 MonTHS At P $<0.05$ RESPECTIVELY

\begin{tabular}{|c|c|c|c|c|c|c|c|c|c|c|c|c|c|c|c|c|c|}
\hline DD & 命 & \multicolumn{2}{|c|}{$\mathrm{NrL}$} & \multicolumn{3}{|c|}{$\begin{array}{l}\text { MAL } \\
{[\mathrm{mm}]}\end{array}$} & \multicolumn{3}{|c|}{$\begin{array}{l}\text { EQD } \\
{[\mathrm{mm}]}\end{array}$} & \multicolumn{3}{|c|}{$\begin{array}{l}\text { PER } \\
{[\mathrm{mm}]}\end{array}$} & \multicolumn{3}{|c|}{$\begin{array}{c}\mathrm{AL} \\
{\left[\mathrm{mm}^{2}\right]}\end{array}$} & \multicolumn{2}{|c|}{$\begin{array}{c}\mathrm{VL} \\
{\left[\mathrm{mm}^{3}\right]}\end{array}$} \\
\hline & & & 6- & & 6- & & & 6- & & & 6- & & & $6-$ & & & \\
\hline $\mathrm{M}$ & & 0 & 12 & 0 & 12 & $\mathrm{~S} / \mathrm{NS}$ & 0 & 12 & $\mathrm{~S} / \mathrm{NS}$ & 0 & 12 & S/NS & 0 & 12 & S/NS & 0 & 6-12 \\
\hline 50 & 1.0 & 8 & 8 & 140 & 109 & NS & 104 & 83 & NS & 390 & 305 & NS & 23 & 140 & NS & 115 & 701 \\
\hline 40 & 2.0 & 4 & 3 & 23 & 19 & NS & 16 & 11 & NS & 61 & 44 & NS & 40 & 27 & NS & 200 & 133 \\
\hline 53 & 2.0 & 11 & 11 & 85 & 86 & NS & 69 & 64 & NS & 237 & 232 & NS & 72 & 64 & NS & 360 & 321 \\
\hline 63 & 2.0 & 9 & 8 & 95 & 198 & S & 70 & 141 & S & 246 & 526 & S & 73 & 229 & $\mathrm{~S}$ & 364 & 1146 \\
\hline 56 & 2.0 & 7 & 7 & 236 & 220 & $\mathrm{~S}$ & 173 & 166 & $\mathrm{~S}$ & 654 & 624 & $\mathrm{~S}$ & 454 & 448 & $\mathrm{~S}$ & 2268 & 2241 \\
\hline 34 & 3.0 & 6 & 4 & 43 & 26 & NS & 36 & 20 & NS & 123 & 69 & NS & 79 & 65 & NS & 397 & 327 \\
\hline 40 & 3.5 & 8 & 8 & 107 & 117 & NS & 85 & 95 & NS & 286 & 321 & NS & 26 & 31 & NS & 128 & 154 \\
\hline 44 & 4.0 & 13 & 10 & 119 & 112 & NS & 86 & 82 & NS & 307 & 291 & S & 40 & 42 & NS & 199 & 212 \\
\hline 50 & 4.0 & 6 & 6 & 26 & 37 & NS & 22 & 27 & NS & 74 & 94 & NS & 31 & 27 & NS & 156 & 134 \\
\hline 40 & 8.0 & 9 & 10 & 176 & 236 & NS & 123 & 173 & NS & 468 & 654 & NS & 165 & 212 & NS & 827 & 1062 \\
\hline Min & 1.0 & 4 & 3 & 23 & 19 & & 16 & 11 & & 61 & 44 & & 23 & 27 & & 128 & 133 \\
\hline Max & 8.0 & 13 & 11 & 236 & 236 & & 173 & 173 & & 654 & 654 & & 454 & 448 & & 2268 & 2241 \\
\hline Med & 3 & 8 & 8 & 101 & 110 & & 77 & 83 & & 266 & 298 & & 56 & 64 & & 362 & 324 \\
\hline IQR & 2 & 3 & 4 & 97 & 127 & & 68 & 114 & & 267 & 432 & & 48 & 181 & & 628 & 908 \\
\hline Aver & 3.3 & 8.1 & 7.5 & 105 & 116 & & 78 & 86 & & 285 & 316 & & 100 & 129 & & 605 & 643 \\
\hline std & 2.1 & 2.6 & 2.6 & 67 & 79 & & 48 & 59 & & 184 & 222 & & 131 & 135 & & 670 & 678 \\
\hline
\end{tabular}

DD: Disease duration for each subject in months, M: Month, EDDS: Expanded disability status scale, IQR: Inter quartile range. NrL: Number of lesions, MAL: Major axis length, EQD: Equivalence diameter, PER: Perimeter, AL: Total lesion area per subject, VL: Total lesion volume per subject, at 0 and 6-12 months respectively.

Twenty-two transaxial slices were acquired with a slice thickness of $5 \mathrm{~mm}$ (section gap of $1 \mathrm{~mm}$ ) and an in-plane spatial resolution of $0.45 \mathrm{~mm} \times 0.45 \mathrm{~mm}$.

\section{B. Manual Delineations and Visual Perception}

All detectable lesions were identified and segmented by an experienced neurologist and confirmed by a neuroradiologist. The neurologist delineated manually (using the mouse) the brain lesions by selecting consecutive points at the visually defined borders between the lesions and the adjacent NAWM on the acquired T2 transverse sections. The manual delineations were performed using a system implemented in MATLAB developed by our group. The measuring points and delineations were saved in order to be used for shape and texture analysis. All sets of manual segmentation measurements were performed by the MS expert in a blinded manner, with respect to identifying the subject, the time-point of the exam, as well as the clinical findings. For each volunteer examined, 10 discrete round regions of interest (with a radius of 30 pixels) were drawn by the neurologist on the NAWM of the obtained T2 images.

The area and the volume of a lesion were calculated as follows:

$$
\begin{aligned}
A & =0.45 \mathrm{~mm} * 0.45 \mathrm{~mm} * \text { NrAreaPixels }\left[\mathrm{mm}^{2}\right] \\
V & =A^{*} 5 \mathrm{~mm}\left[\mathrm{~mm}^{3}\right]
\end{aligned}
$$

\section{Texture Analysis}

In this study the following texture features, and shape parameters were extracted from all MS lesions detected and segmented, as well as from all the segmented healthy brain areas [5], [8], [10]:

(i) Statistical Features (SF): 1) standard deviation $(\sigma)$. (ii) Spatial Gray Level Dependence Matrices (SGLDM) as proposed by Haralick et al. [10]: 1) Contrast, 2) Sum of squares: variance, 3) Inverse difference moment, 4) Sum average, 5) Sum variance, 6) Sum entropy, 7) Entropy, 8) Difference variance, and 9) Difference entropy. Each feature was computed using a distance of one pixel. Also for each feature the mean values and the range of values were computed, and were used as two different feature sets. (iii) Shape Parameters: The following shape parameters were calculated from the segmented MS lesions. 1) $\mathrm{X}-$ coordinate maximum length, 2) $\mathrm{Y}$-coordinate maximum length, 3) area, 4) perimeter, 5) perimeter ${ }^{2} /$ area, 6) eccentricity, 7) equivalence diameter, 8) major axis length, 9) minor axis length, 10) centroid, 11) convex area, and 12) orientation, where the most significant are shown.

As data were not normally distributed or had unequal variances, the Mann-Whitney rank sum test, which calculates the difference between the sum of the ranks of two different independent samples, was used in order to 
identify if for each set of measurements a significant difference (S) or not (NS) exists between all extracted texture features, with a confidence level of $95 \%$.

The Wilcoxon matched pairs rank sum test which calculates the difference between the sum of the ranks of two dependent samples, was also used in order to identify if for each set of measurements a significant difference (S) or not (NS) exists, at $p<0.05$. Furthermore, histograms of the lesions were used in order to demonstrate the difference of the grayscale values between 0 and 6-12 months.

\section{RESULTS}

Figure 1 displays regions of interest (ROIs) of segmented lesions from the MRI scans at 0 and 6-12 months, and their corresponding histogram distribution. The median (IQR) of the lesions at 0 and 6-12 months were median=122 $(\mathrm{IQR}=16.4)$ vs median=118 $(\mathrm{IQR}=24)$ respectively.

Table I presents the shape features statistical analysis for the lesions detected within each subject at 0 and 6-12 months. It is shown that there was a slight increase in the median (IQR) of the lesions from all subjects between 0 and 6-12 months, for the major axis length (MAL) median=101 ( $\mathrm{IQR}=97)$ vs median=110 (IQR=27), equivalence diameter median=77 (IQR=68) vs median=83 (IQR=114), and perimeter median=266 (IQR=267) vs median $=298$ $(\mathrm{IQR}=432)$ respectively.

Table II presents the shape feature statistical analysis for all lesions for all subjects at 0 and 6-12 months. It is shown that there was no significant difference between the features at 0 and 6-12 months respectively.

Table III presents the texture features median (IQR) extracted from healthy (NAWM) tissue, as well as from all lesions at 0 and 6-12 months. The Man Whitney test performed between the features extracted showed that most of the features are significantly different $(\mathrm{p}<0.05)$ between: i) normal tissue and lesions at 0 months, ii) between normal tissue and lesions at 6-12 months, and iii) between lesions at 0 and 6-12 months.

Figure 2 gives the box plots of the contrast feature. It shows clearly the increase between normal and lesions at 0 and 6-12 months.

\section{Discussion}

The objective of this study was to assess the value of MRI shape and texture analysis in the study of MS brain lesions both in differentiating between normal and abnormal tissue and in assessing disease progression. Texture features and descriptive measures were extracted and investigated, based on statistical measures, and univariate statistical analysis, from the manually segmented MS lesions of 10 subjects, and from healthy WM areas, in an attempt to quantify pathological changes that occur in MS. The study showed that: i) there was no significant difference (SD) between the shape features extracted from the lesions of 0 and 6-12

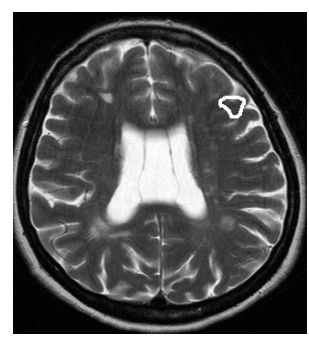

a)

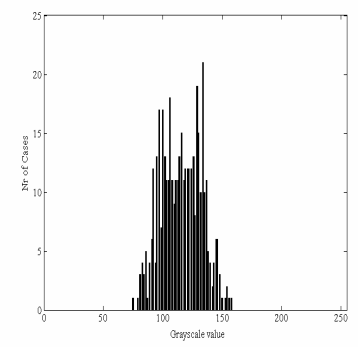

c) 0 months histogram

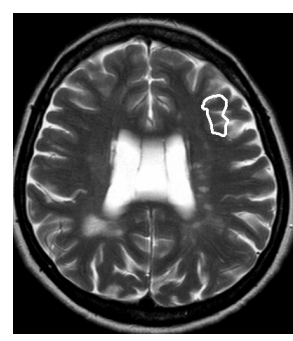

b)

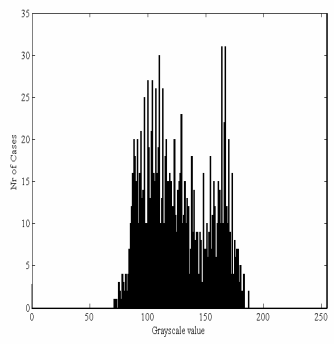

d) 6 months histogram
Fig. 1. a) ROIs of MR image of the brain from a patient with MS lesion in the gray matter at 0 and 6-12 months. Histograms from the lesion at 0 and 6-12 months in c) and d) respectively.

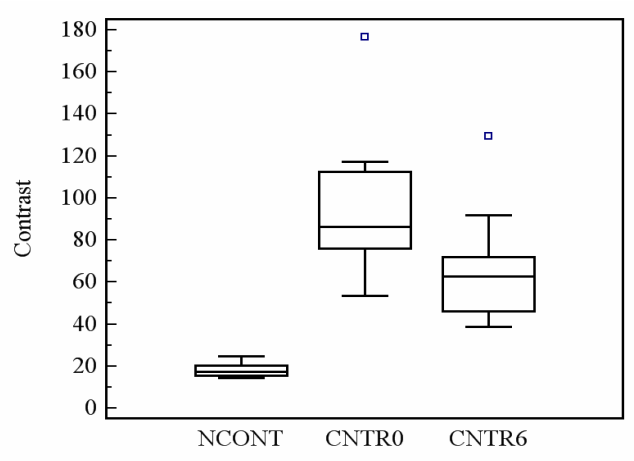

Fig. 2. Box plots of contrast for normal tissue (NCONT) and lesions at 0 (CNTR0) and 6-12 (CNTR6) months respectively.

months respectively, ii) there was a SD between all of the texture features extracted from the normal tissue and texture features extracted from the lesions at 0 and 6-12 months with the exception of entropy (see Table III), and iii) there was a SD for some features (contrast, IDM, sum average, $\mathrm{DV}, \mathrm{DE})$ extracted from the lesions at 0 and 6-12 months.

Table II showed that there was no SD (only a slight change) of shape features between lesions of 0 and 6-12 months. Further research with more subjects is required for computing shape features that may provide information for the longitudinal monitoring of these lesions. In [2] it was shown that new lesions and increase in disease volume is very weakly associated $(\rho=0.13-0.23)$ with increase in disability (EDSS). On the other hand a simple correlation of the progression of lesions in relation to the EDSS is extremely unlikely as documented in [3].

Table III showed that some of the texture features are 
TABLE II

Shape FeAture StatisticAl AnAlysis For All Lesions For All SubJECTS AT 0 AND 6-12 MONTHS. WilCOXON RANK SUM TEST Shows With S AND NS the Features That ARE AND ARE Not SIGNIFICANTLY DIFFERENT FOR 0 AND 6-12 MONTHS AT $\mathrm{P}<0.05$ RESPECTIVELY

\begin{tabular}{lc}
\hline \hline \multicolumn{1}{c}{ Features } & 0 vs 6-12 months \\
\hline Area & NS $(0.58)$ \\
Volume & NS $(0.85)$ \\
Perimeter & NS $(0.92)$ \\
Eccentricity & NS $(0.56)$ \\
Equivalence Diameter & NS $(0.98)$ \\
Major Axis Length & NS $(0.92)$ \\
Minor Axis Length & NS $(0.92)$ \\
\hline \hline
\end{tabular}

TABLE III

TEXTURE FEATURES (MEDIAN-IQR) FOR OF LESIONS For AlL SubJECTS AT 0 AND 6-12 MONTHS. THE MAN WHITNEY RANK SUM TEST SHOWs With (S) SigNifiCANTLY AND (NS) NON SigNifiCANTLY DifFERENT FEATURES BETWEEN NORMAL TISSUE AND LESIONS AT 0 AND 6-12 MONTHS.

\begin{tabular}{|c|c|c|c|c|c|c|}
\hline $\begin{array}{l}\text { Texture } \\
\text { Feature }\end{array}$ & $\begin{array}{l}\text { Normal } \\
\text { Tissue }\end{array}$ & $\begin{array}{c}\text { Lesions at } \\
0 \\
\text { months }\end{array}$ & $\begin{array}{c}\text { Lesions at } \\
6-12 \\
\text { months }\end{array}$ & $\begin{array}{l}Z \\
\text { L } \\
0\end{array}$ & $\begin{array}{l}Z \\
u \\
\infty \\
a \\
i \\
N\end{array}$ & $\begin{array}{l}0 \\
\dot{s} \\
0 \\
\frac{1}{N}\end{array}$ \\
\hline $\begin{array}{l}\text { Stand. Dev. } \\
\text { Median } \\
\text { Contrast } \\
\text { SOSV } \\
\text { Entropy } \\
\text { IDM } \\
\text { Sum Aver. } \\
\text { Sum Var. } \\
\text { DV } \\
\text { DE } \\
\end{array}$ & $\begin{array}{l}5.3(0.56) \\
71(7.2) \\
23(4.3) \\
28(5.2) \\
5.2(0.3) \\
0.2(0.1) \\
144(14) \\
88(15) \\
8.4(1.5) \\
2.3(0 .) \\
\end{array}$ & $\begin{array}{l}17.4(4.4) \\
127(8.2) \\
87(46) \\
326(176) \\
5.27(0.4) \\
0.15(0.0) 1 \\
257(0.3) \\
1219(46) \\
30(18) \\
2.7(0.05) \\
\end{array}$ & $\begin{array}{l}15(2.5) \\
116(16) \\
63(15) \\
237(86) \\
5.25(0.5) \\
0.16(0.1) \\
236(0.2) \\
884(30) \\
22(6) \\
2.6(0.01) \\
\end{array}$ & $\begin{array}{c}\mathrm{S} \\
\mathrm{S} \\
\mathrm{S} \\
\mathrm{S} \\
\mathrm{NS} \\
\mathrm{S} \\
\mathrm{S} \\
\mathrm{S} \\
\mathrm{S} \\
\mathrm{S} \\
\end{array}$ & $\begin{array}{c}\mathrm{S} \\
\mathrm{S} \\
\mathrm{S} \\
\mathrm{S} \\
\mathrm{NS} \\
\mathrm{S} \\
\mathrm{S} \\
\mathrm{S} \\
\mathrm{S} \\
\mathrm{S} \\
\end{array}$ & $\begin{array}{c}\text { NS } \\
\text { NS } \\
\text { S } \\
\text { NS } \\
\text { NS } \\
\text { S } \\
\text { S } \\
\text { NS } \\
\text { S } \\
\text { S } \\
\end{array}$ \\
\hline
\end{tabular}

SOSV: Sum of squares variance, IDM: Inverse difference moment, DV Difference variance, DE: Difference entropy, IQR: Inter quartile range

sensitive to the progression of the MS disease. It was shown that texture features such as contrast, difference variance, difference entropy, increase with the onset of the disease when compared with their corresponding normal tissue values, whereas with the progression of the disease to 6-12 months they decrease when compared with the corresponding tissue values at 0 months (see also Fig. 2).

Various studies have been performed in order to establish a relationship between the various gray levels and texture features [3]-[6]. It was shown in [3], that histograms can characterise changes between MS lesions and NAWM. It was also shown in this study that texture features and histograms may be used in discriminating between lesions of normal and abnormal tissue. In [5] significant differences in texture were also found between normal and MS patients in the spinal cord. The increase of these texture features suggests that the lesions texture in MS patients is less homogeneous and more complex than in normal subjects [5]. This loss in homogeneity may be a result of some pathological processes, including demyelination, gliosis, inflammation, axonal loss, or changes in water content and leading to a less uniform MR signal intensity. Similar findings were also reported in this study where it is shown that texture features increase with the onset of the MS disease when compared to normal tissue see Table III).

In another study [7], it was shown that texture analysis can achieve high classification accuracy $(>=90 \%)$ in tissue discrimination between MS lesions and NAWM.

It should be noted that shape and texture analysis as presented in this study is dependent from i) acquisition parameters, ii) the quality assessment of the MRI device, and iii) the methods of image reconstruction and processing [4]. Furthermore, as documented in [4], an open remaining question deals with the correspondence between texture analysis and histologic parameters as the voxel resolution is very large if compared to histologic structures.

The findings of this study are preliminary and are based on a small number of subjects. Further research work with more subjects is required for computing shape and texture features that may provide information for differentiating between normal tissue and MS lesions as well as for longitudinal monitoring of these lesions. In addition the proposed methodology can be used for the assessment of subjects at risk of developing future neurological events. The extracted features can also offer additional information of undiagnosed lesions

\section{REFERENCES}

[1] F. Fazekas, F. Barkof, M. Filippi, et al, "The contribution of magnetic resonance imaging to the diagnosis of multiple sclerosis", Neur., vol. 53, pp. 448-456, 1999.

[2] M. Filippi, D.W. Paty, L. Kappos, F. Barkhof, D.A. Compston, A.J. Thompson, G.J. Zhao, C.M.Wiles, W.I. McDonald, D.H. Miller, "Correlations between changes in disability and T2-weighted brain MRI activity in multiple sclerosis : a follow-up study", Neurology, vol. 45 , pp. $255-260,1995$.

[3] J. Dehmeshki G.J. Barker, P.S. Tofts, "Classifications of disease subgroups and correlation with disease severity using magnetic resonance imaging whole-brain histograms: Application to magnetisation transfer ratios and multiple sclerosis", IEEE Trans. Med. Imag., vol. 21, no. 4, pp. 320-331, 2002.

[4] S. Herlidou-Meme, J.M. Constans, B. Carsin, D. Olivie, P.A. Eliat et al., "MRI texture analysis on texture test objects, normal brain and intracranial tumours", Mag. Res. Imag., vol. 21, pp. 989-993, 2003.

[5] J.M. Mathias, P.S. Tofts, N.A. Losseff, "Texture analysis of spinal cord pathology in multiple sclerosis", Magn, Res. in Med., vol. 42, pp. 929-935, 1999.

[6] O. Yu, Y Mauss, G. Zollner, I.J. Namer, J. Chambron, "Distinct patterns of active and non-active plaques using texture analysis of brain NMR images in multiple sclerosis patients: Preliminary results", Magn. Reson. Imag., vol. 17, no. 9, pp. 1261-1267, 1999.

[7] J. Zhang, L. Wang, L. Tong, "Feature reduction and texture classification in MRI-Texture analysis of multiple sclerosis", IEEE/ICME Conf. Complex Med. Eng., pp. 752-757, 2007.

[8] P.A. Freeborough, N.C. Fox, "MR Image texture analysis to the diagnosis and tracking of Alzheimer disease", "IEEE Trans. Med. Imag., vol. 17, no. 3, pp. 475-479, 1998.

[9] A.J. Thompson, J.C Hobart, "Multiple sclerosis: assessment of disability and disability scales", J. Neurol., vol. 254, no.4, pp. 189196, 1998

[10] R.M. Haralick, K. Shanmugam, and I. Dinstein, "Texture Features for Image Classification", IEEE Trans. Systems, Man., and Cybernetics, vol. SMC-3, pp. 610-621, Nov. 1973. 